Effects of changes Discontinuing the use of midazolam sedation has prevented children undergoing a stressful procedure that was unsuccesful in $82 \%$ of cases. This has improved patient care in the form of reducing delays in diagnosis and lowering parental anxiety. It has also saved the trust c. $£ 70,000$ per year in failed scans and referrals to $\mathrm{BCH}$.

Lessons learnt This project has shown me that using a structured approach when considering a problem makes it easier to change systems/processes. Our department knew that midazolam wasn't particularly successful but when you present that as a $82 \%$ failure rate it makes things a lot clearer. Forming an MDT to try to find a solution was also of huge benefit as it very quickly became clear that the radiology department shared our frustrations regarding failure of sedation. This in turn has enabled us to move forward with development of our own GA scan service quite quickly.

Message for others If you feel there is a problem/issue in your department analyse it in a structured way and try to form a solution to the issue rather just presenting a problem

If an MDT needs to be involved ensuring that there are clear goals identified is very important so as to avoid being sidetracked.

\section{G569(P) PATIENT SAFETY IMPROVEMENT PROJECT. PRESCRIPTION OF INTRAVENOUS FLUIDS FOR ADMINISTRATION IN CHILDREN}

S Deepak, T Kwok, T Tinklin. Paediatric Trainee, Royal Derby Hospital, Derby, UK

\subsection{6/archdischild-2015-308599.518}

Context The prescription of IV fluids is a commonplace task on the paediatric ward and its importance is often underestimated. National Patient Safety Agency (NPSA) has issued an alert in 2007 advising clinicians about the risk of hyponatraemia in children receiving intravenous (IV) fluids. Accurate prescription of IV fluids and careful monitoring are crucial in preventing fluid induced hyponatraemia in children.

Hence, a local IV fluid prescription guideline was developed and a patient safety improvement project was set up to ensure that the guideline is adhered to.

Problem A prospective audit was performed in November 2012, looking at all children who were admitted to a busy district general children hospital and prescribed IV fluids over a 2 week period. Prescription of IV fluid rate, type of fluid used, potassium content as well as monitoring of patient's renal function, fluid balance and daily weight were audited.

Assessment of problem and analysis of its causes The results of this audit show that type of fluid, rate of fluid and daily monitoring of UEs are done relatively well. Weight monitoring and fluid balance was poorly done.

Intervention We took few actions to improve the outcome.

Further education of junior doctors, nurses and allied health care professionals was provided.

Fluid prescription chart was updated to act as a visual cue and to enable accurate calculation of fluid rate and renal function monitoring.

Strategy for change Our strategy to improve the compliance to the standards was by focussing on the targeted education package for both medical and nursing team.
We designed an IV fluid teaching booklet with assessments to be carried out by all junior doctors during the departmental induction followed by a seminar every few months.

Awareness regarding the standards was created among nursing colleagues.

Measurement of improvement A similar prospective reaudit was performed in November 2013 on all children admitted over a 2 week period in the same hospital.

Effects of changes The prescription of type of fluid, rate of fluid and daily monitoring of UEs are done relatively well. Prescription of potassium containing fluids, monitoring of daily weights and fluid balance which were badly done on the initial audit with compliance rate of $40 \%, 40 \%$ and $58 \%$ respectively improved to $71 \%, 71 \%$ and $61 \%$ during the reaudit.

Lessons learnt This project highlights the importance of education and enhanced training for staff. We are highlighting the results to all clinician as well as nursing staff during departmental meetings to raise awareness and identify solutions to barriers identified on a regular basis.

Message for others Targeted staff education and training are paramount important in improving the quality of care.

These can be cost effective and by effective organisation, can successfully implement locally.

\section{G570(P) PROJECT PLAY - MEDICAL STUDENTS PROVIDING OUT OF HOURS PLAY FOR PAEDIATRIC PATIENTS}

${ }^{1} \mathrm{H}$ Pearson, ${ }^{1} \mathrm{~L}$ Harlin-Bethel, ${ }^{2} \mathrm{~T}$ Shea. ${ }^{1}$ School of Medicine and Dentistry, Barts and the London, London, UK; ${ }^{2}$ Play Specialist, Barts and the London, London, UK

\subsection{6/archdischild-2015-308599.519}

Context This study examines the effectiveness of Project Play; a collaboration between medical students and health play specialists in order to provide an out of hours play service at The Royal London Hospital (RLH).

Problem There is no out of hours play for children, and there is not enough clinical attachment time for medical students on paediatrics.

Assessment of problem and analysis of its causes The Play Department is currently only able to provide patients with a service Monday to Friday 9-5 pm. The patients need to have access to therapeutic play outside of these hours, however due to staffing we are unable to provide this service.

Students have expressed a high level of interest in paediatrics and a recurrent comment is that there is not enough time in the core module provided for paediatric clinical attachments.

Dissatisfaction is show in this quote from the head of the Human Development module: "Although students have expressed a high level of satisfaction with the Paediatric modules in their Human Development course, a recurrent comment is that there is not enough time in the core module provided for all students. Ours is one of the shortest clinical attachments of UK medical schools."

Intervention All volunteers were required to attend a training day. Then, two volunteering session a week were initiated in October 2014. These ran 6-8 pm on Tuesday and Thursday evenings. Tuesday sessions were aimed at children aged 3-12 years, Thursday 12-18 years. The number of volunteers at each session ranged from 4 to 7 . There is one lead volunteer from the committee at each session. 